\title{
PERIODIC ORBITS OF HYPERBOLIC DIFFEOMORPHISMS AND FLOWS ${ }^{1}$
}

BY MICHAEL SHUB

\author{
Communicated by J. Moser, July 31, 1968
}

Artin and Mazur in [1] proved that a dense subset of the $C_{\text {- }}$ endomorphisms of a compact differentiable manifold satisfy an exponential growth condition on their isolated periodic points, and they defined a $\zeta$-function which for these endomorphisms has a positive radius of convergence. In [2] and [3] K. Meyer gave a simple proof that hyperbolic diffeomorphisms and flows of Smale [4] which are $C^{2}$ have exponential growth. It is the purpose of this note to give an even simpler proof of Meyer's theorems in a $C^{1}$ setting. Since the hyperbolic diffeomorphisms and flows are not dense [5] these results are a long way from including the results of [1].

Let $M$ be a compact differentiable manifold; let $f \in \operatorname{Diff}(M)$ be a $C^{1}$ diffeomorphism, and let $N_{m}(f)$ be the number of periodic points of $f$ of period $m$.

THEOREM 1. Let $f$ satisfy Axiom A of $[4,1.6]$, then there exist constants $c$ and $k$ such that $N_{m}(f) \leqq c k^{m}$.

Proof. $f$ is expansive [4, I.8.7], i.e., $\exists \epsilon>0$ such that given $x, y$ distinct periodic points of $f \exists n \in Z$ such that $d\left(f^{n}(x), f^{n}(y)\right) \geqq \epsilon$. Since $f$ is $C^{1}$ it is Lipshitz. Let its Lipshitz constant be $k$ which we may choose $>1$. If $x$ and $y$ are both of period $p$ we may choose $n$ in $0 \leqq n<p$ and have $d(x, y) \geqq \epsilon / k^{p-1}$ by expansiveness. Thus there exists a constant $c$ such that $N_{p}(f) \leqq c V(M)\left(2 k^{p-1} / \epsilon\right)^{\operatorname{dim} M}$ where $V(M)$ is the volume of $M$.

Let $\Phi=\left\{\phi_{t}\right\}$ be a one parameter group acting on $M$, arising from a $C^{1}$ vector field $X$. Let $N_{\tau}(\Phi)$ be the number of closed orbits of $\Phi$ of period less than or equal to $\tau$.

Theorem 2. Let $\Phi$ satisfy Axiom $\mathrm{A}^{\prime}$ of $[4,5.1]$, then there exist constants $c$ and $k$ such that $N_{\tau}(\Phi) \leqq c e^{k r}$.

Since the closed orbits are uniformly bounded away from the singularities, which are finite in number, $\Omega_{c}$ the complement of the singularities in $\Omega$, is compact. Every point $z$ in $\Omega_{c}$ has a flow box

1 This work was done while the author was partially supported by National Science Foundation Grant No. 6868. 
neighborhood, $V_{z}$, such that $V_{z}$ has a cross section $X_{z}$; and for some $\delta, V_{z}=\bigcup_{x \in X_{z},|t|<\delta} \phi_{t}(x)$ where $\delta$ is independent of $z$. Denote by $\pi_{z}: V_{z} \rightarrow X_{z}$ the map which takes $\phi_{t}(x)$ to $x$ for $|t|<\delta$ and $x \in X_{z}$. The $V_{z}$ and $t$ may be chosen so that if $x, y \in X_{z}$ and $\phi_{t}(x), \phi_{t}(y) \in X_{w_{1}}$; $\phi_{-t}(x), \phi_{-t}(y) \in X_{w_{2}}$ then either $\pi_{w_{1}} \phi_{t}$ or $\pi_{w_{2}} \phi_{-t}$ increases their distance by a factor of $k_{1}>1 ; k_{1}$ independent of $z$.

Now cover $\Omega_{c}$ by a finite number of $V_{z} ; V_{1}, V_{2}, \cdots, V_{n}$. Note that if $x \in X_{i}$ and $\phi_{t}(x) \in V_{j}$ then $\pi_{i} \phi_{-t} \pi_{j} \phi_{t}(x)=x$, and similarly for $\phi_{-t}$. Define an invariant sequence of a closed orbit $\alpha \subset \Omega_{c}$ as a sequence $a_{1} \cdots a_{m}$ such that $a_{i}=1, \cdots, n ; a_{1}=a_{m}$ and there exist $x_{a_{i}} \in X_{a_{i}}$ where $x_{a_{1}}=x_{a_{m}}, \pi_{a_{i}} \phi_{t}\left(x_{a_{i-1}}\right)=x_{a_{i}}$ and $\pi_{a_{i-1}} \phi_{-t}\left(x_{a_{i}}\right)=x_{a_{i-1}}$. It is clear from the definition and the choice of the $V_{i}$ that no two distinct closed orbits may have the same invariant sequence. We will show that $\exists c>0$ such that a closed orbit of period $\leqq \tau$ has an invariant sequence of length at most ncr+1, and thus the number of closed orbits of period $\leqq \tau$ is less than or equal to $n^{n e r+2}=n^{2} e^{\text {re }} \log n$.

To get the invariant sequence, $\exists c>0$ such that if $\alpha$ is a closed orbit of period $\leqq \tau$ then $\alpha$ intersects $X_{i}$ for any $i$ in at most $c \tau$ points, $c$ is independent of $\tau$. So there are at most $n c \tau$ intersections of $\alpha$ with all the $X_{i}$. Let $x_{0}$ be one of these. Define $x_{i}$ inductively by $x_{i+1}=\pi_{j} \phi_{t}\left(x_{i}\right)$ where $\phi_{t}\left(x_{i}\right) \in V_{j}$ for some $j . x_{k}$ must equal $x_{m}$ for some $k, m \leqq n c \tau$ and $k \neq m$.

Of course, this proof would also work in the diffeomorphism case. The construction of the corresponding $V_{i}$, however, essentially gives the proof of expansiveness which was shown to me by Smale.

These theorems, of course, have relevance for the convergence of the zeta functions. For a discussion of this see [3] and [4].

\section{REFERENCES}

1. M. Artin and B. Mazur, On periodic points, Ann. of Math. (2) 81 (1965), 82-99.

2. K. R. Meyer, Periodic points of diffeomorphisms, Bull. Amer. Math. Soc. 73 (1967), 615-617.

3. - On the convergence of the zeta function for flows and diffeomorphisms, J. Differential Equations (to appear).

4. S. Smale, Differentiable dynamical systems, Bull. Amer. Math. Soc. 73 (1967), 747-817.

5. R. Abraham and S. Smale, Non-genericity of \&-stability (to appear).

Brandeis University, Waltham, Massachusetts 02154 